\title{
Trajectory Reconstruction of Orion Ground Test Article Water Impact Tests
}

\author{
Rafael A. Lugo*, Christopher D. Karlgaard ${ }^{\dagger}$ \\ Analytical Mechanics Associates, Inc., Hampton, VA 23666 \\ James M. Corliss $*$ \\ NASA Langley Research Center, Hampton, VA 23681 \\ Gregory J. Vassilakos ${ }^{\S}$ \\ Science and Technology Corporation, Hampton, VA 23666 \\ and \\ Stephen D. Mark ${ }^{* *}$ \\ Science and Technology Corporation, Hampton, VA 23666
}

The Orion Crew Module is a component of NASA's Multi-Purpose Crew Vehicle that will be used for future missions to low Earth orbit and beyond. Ten water impact tests of the Orion Ground Test Article (GTA) were conducted at the Hydro Impact Basin at NASA Langley Research Center in 2016 and were designed to provide data for the validation of the LS-DYNA model used to determine the Crew Module structural loads during ocean splashdown, and the determination of an acceptable Model Uncertainty Factor to apply to simulation results used to drive the design. Post-test data obtained from the onboard sensors were used to reconstruct the GTA trajectories both before and after water impact. Results from one vertical test and two swing tests are presented and compared to videos taken for each test.

\section{Nomenclature}

a $=$ body acceleration vector

ALE $\quad=$ Arbitrary Lagrangian-Eulerian

$\mathrm{CG}=$ center of gravity

EFT $=$ Exploration Flight Test

$\mathrm{EM} \quad=$ Exploration Mission

EVE $=$ Exploration Visual Environment

GTA $=$ Ground Test Article

HIB $=$ Hydro Impact Basin

MPCV = Multi-Purpose Crew Vehicle

NED $\quad=$ North-East-Down

NewSTEP $=$ New Statistical Trajectory Estimation Program

SLS = Space Launch System

ST $=$ Swing Test

STEP $=$ Statistical Trajectory Estimation Program

VT $=$ Vertical Test

\footnotetext{
* Aerospace Engineer, Atmospheric Flight and Entry Systems Branch, NASA Langley Research Center, MS 489, AIAA Member $\dagger$ Supervising Engineer, Atmospheric Flight and Entry Systems Branch, NASA Langley Research Center, MS 489, AIAA Senior Member

¥ Chief Engineer, Orion GTA Water Impact Tests, Atmospheric Flight and Entry Systems Branch, NASA Langley Research Center, MS 489

$\S$ Senior Dynamics Analyst, Structural Dynamics Branch, NASA Langley Research Center, MS 230

** Senior Dynamics Analyst, Structural Dynamics Branch, NASA Langley Research Center, MS 495
} 


$\begin{array}{ll}\text { WIT } & =\text { Water Impact Test } \\ X, Y, Z & =\text { inertial axes } \\ x, y, z & =\text { body axes } \\ \omega & =\text { body rate vector }\end{array}$

\section{Introduction}

The Multi-Purpose Crew Vehicle (MPCV) is a NASA spacecraft designed to transfer crews to low Earth orbit (LEO) and beyond. The Orion Crew Module (CM) is the component of the MPCV in which the crew live during the mission, and is also the spacecraft that returns the crew to Earth, ending with an ocean splashdown at the end of each mission. An uncrewed MPCV was first flown to Earth orbit during the Exploration Flight Test 1 (EFT-1) mission in December 2014. ${ }^{1}$ An uncrewed MPCV is scheduled to be flown on the Space Launch System (SLS) for Exploration Mission 1 (EM-1) in 2019, and a crewed MPCV is scheduled to be flown on EM-2 in the early 2020s. ${ }^{2,3}$ Figure 1 shows an illustration of the Orion CM as it would look during EM-1.

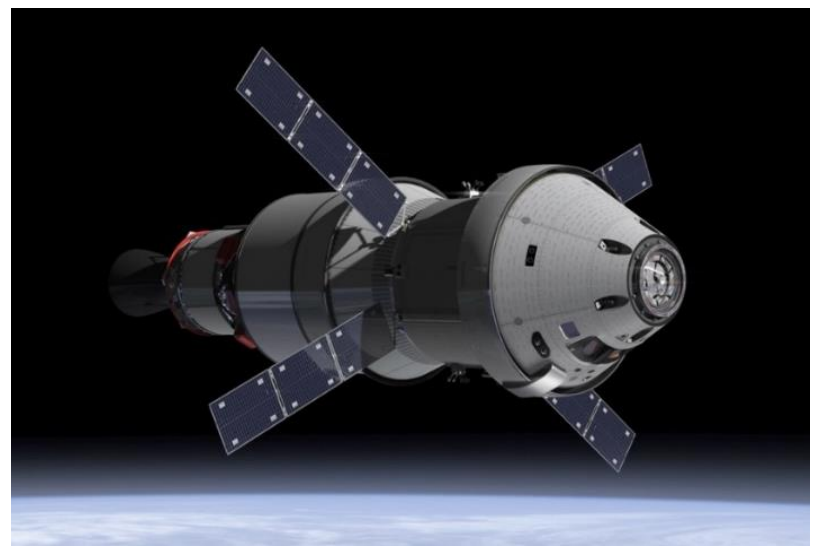

Figure 1. MPCV as it will appear during EM-1. The conical Orion Crew Module is at right, atop the European Service Module (ESM) with deployed solar panel arrays, and Interim Cryogenic Propulsion System (ICPS). Image Credit: www.nasa.gov

To support the performance analysis of the CM structure, a prototype Orion Ground Test Article (GTA) outfitted with the Orion heatshield flown on EFT-1 was tested at the Hydro Impact Basin (HIB) at NASA Langley Research Center. Ten water impact tests (WIT) designed to characterize the structural loads were conducted from April to September of 2016. Post-test data obtained from the onboard sensors were used to reconstruct the GTA trajectories both before and after water impact. The resultant trajectories were then compared to video taken of each test and photogrammetric reconstructions, as well as simulated trajectories from LS-DYNA. ${ }^{4,56}$

\section{Overview of Water Impact Tests}

The GTA WIT were conducted to validate the LS-DYNA model that the Orion Project uses to determine the CM structural loads when it splashes down in the ocean at the end of each mission. Ocean splashdown produces the peak loads that a large portion of the CM structure experiences throughout Orion's planned missions. A validated loads model is critical to ensuring that the CM is designed to withstand the ocean landing and protect the crew. The LSDYNA model is comprised of three primary components: 1) a finite element model of the CM/GTA structure; 2) a finite element model representing the ocean water and the air above it; and 3) parameters that govern the fluidstructure-interaction between the water and the CM/GTA structure. The purpose of the GTA WIT effort was to demonstrate that these three model elements work together in the landing simulation to produce accurate landing loads.

\section{A. Orion Ground Test Article}

The GTA is a flight-like prototype of the Orion CM structure and is shown in Figure 2. It was integrated with the post-flight heatshield from Exploration Flight Test 1 (EFT-1) to form the complete test article for the water impact tests. The tests were designed to represent some of the most stressing landing scenarios that the Crew Module could experience, including landings with only two of the three descent parachutes deployed and ascent abort landings in 
high winds. Ten tests, of both vertical (VT) and swing (ST) types, were conducted to span these different landing scenarios, with 530 channels of load data acquired during each test. The acquired data included strains and deflections throughout the GTA structure, water pressures on the wetted surfaces, and GTA accelerations and rotation rates. These data were used to validate the LS-DYNA model and to conduct a rigid-body dynamics reconstruction of each test.

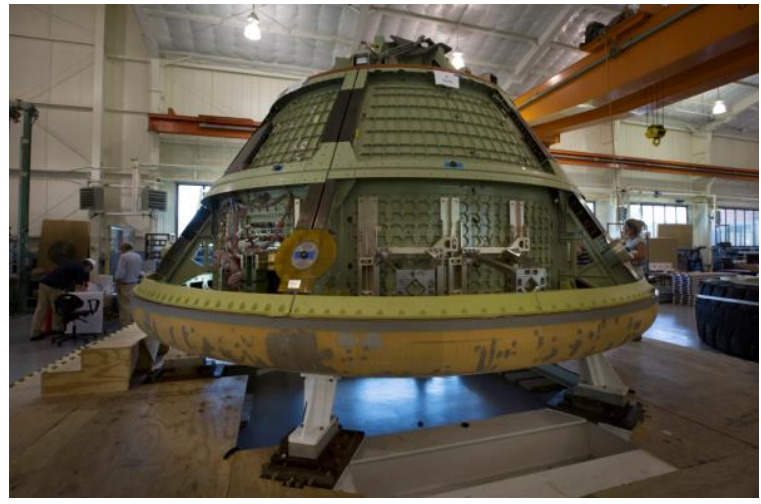

(a)

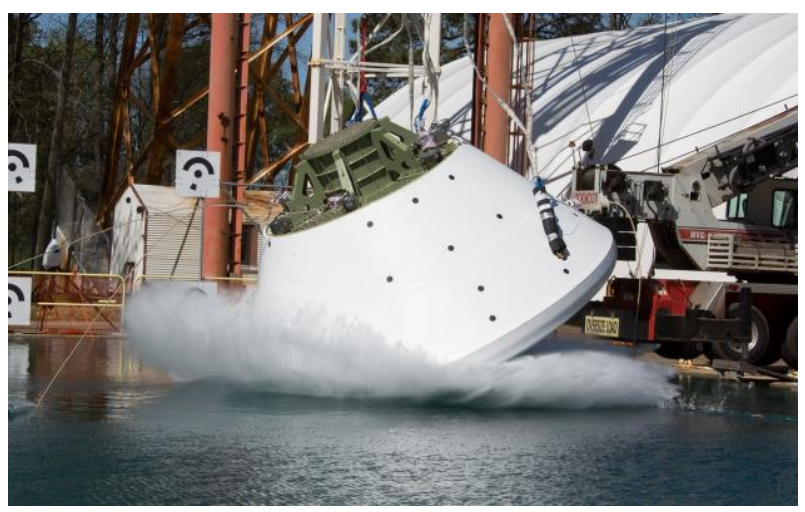

(b)

Figure 2. Orion GTA (a) prior to backshell fixture and (b) during WIT. Image Credits: NASA.gov

\section{B. Coordinate Systems}

The North-East-Down (NED) frame, fixed to the surface of a flat, non-rotating Earth so that it is inertial, has the $x$-axis pointing North, the $y$-axis pointing East, and the $z$-axis pointed down into the ground. The body frame used in the analysis was fixed to the GTA with the origin at the GTA center of gravity (CG), the $x$-axis pointing towards the center of the heatshield, the $z$-axis pointing towards the centerline between the viewports, and the $y$-axis completing the right-hand frame. These frames are illustrated in Figure 3, with the body frame shown at left in black and the NED frame shown at right in red.

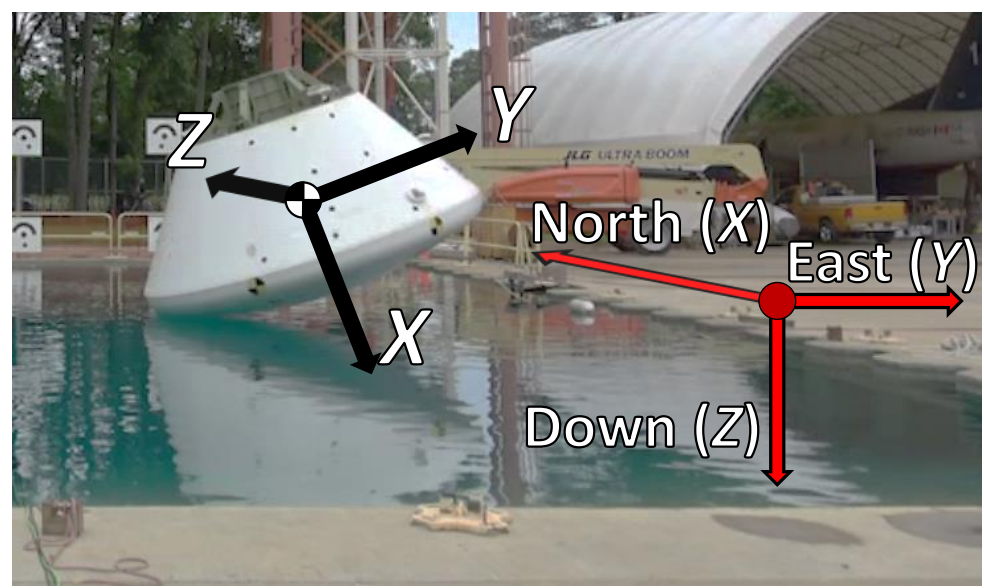

Figure 3. Coordinate systems. At left in black, body coordinate system attached to GTA. At right in red, inertial NED coordinate systems.

\section{Flight Data}

Although the GTA was equipped with several accelerometers and gyroscopes, for the purposes of trajectory reconstruction the only acceleration data used were those obtained from the three single-axis accelerometers located on the "seat backbone". Rate data from three tri-axial gyroscopes were averaged with weights based on the manufacturer's published full scale noise values. Information on the sensors used in the trajectory reconstruction is listed in Table 1. The sensor direction is the GTA body frame shown in Figure 3. Note that although the sensors were not specifically designed for use in trajectory reconstruction, the data were for the most part sufficient to perform trajectory reconstructions for the first few seconds after water impact. 
Table 1. GTA onboard sensors used in trajectory reconstruction.

\begin{tabular}{ccccc}
\hline \hline Sensor & Type and Direction & Location & Range & Noise (full scale) \\
\hline MS 3801A-500 & Accelerometer (-X) & Seat Backbone & $\pm 500 \mathrm{~g}$ & $0.5 \%$ (assumed) \\
MS 3801A-100 & Accelerometer (+Y) & Seat Backbone & $\pm 100 \mathrm{~g}$ & $0.5 \%$ (assumed) \\
MS 3801A-500 & Accelerometer (+Z) & Seat Backbone & $\pm 500 \mathrm{~g}$ & $0.5 \%$ (assumed) \\
DTS ARS3 PRO-300 & Gyroscope & Seat Backbone & $\pm 300 \% \mathrm{~s}$ & $0.6 \%$ \\
DTS ARS3 PRO-300 & Gyroscope & Access Tunnel Lid & $\pm 300 \% \mathrm{~s}$ & $0.6 \%$ \\
DTS 6DX PRO-1500 & Rate sensor & Access Tunnel Lid & $\pm 1500 \% \mathrm{~s}$ & $5 \%$ (assumed) \\
\hline
\end{tabular}

The data consists of $20,000 \mathrm{~Hz}$ engineering unit accelerations and angular rate measurements expressed in the body frame, provided by the onboard accelerometers and rate gyroscopes and smoothed using a $200 \mathrm{~Hz}$ low-pass filter. The data used in the reconstruction process were smoothed and transformed to the GTA CG, and are shown in Figure 4, with $0 \mathrm{~s}$ being the time of impact for each WIT. As will be discussed in greater detail later in this paper, during Swing Test 7 (ST7) the GTA impacted the water at a steeper angle than expected, causing the GTA to rise up faster and completely exit the water before experiencing a secondary impact. This event can be seen in the data between approximately $1.5 \mathrm{~s}$ and $2.8 \mathrm{~s}$, where the accelerations go to zero and the rates show a constant slope, consistent with a vehicle in freefall.
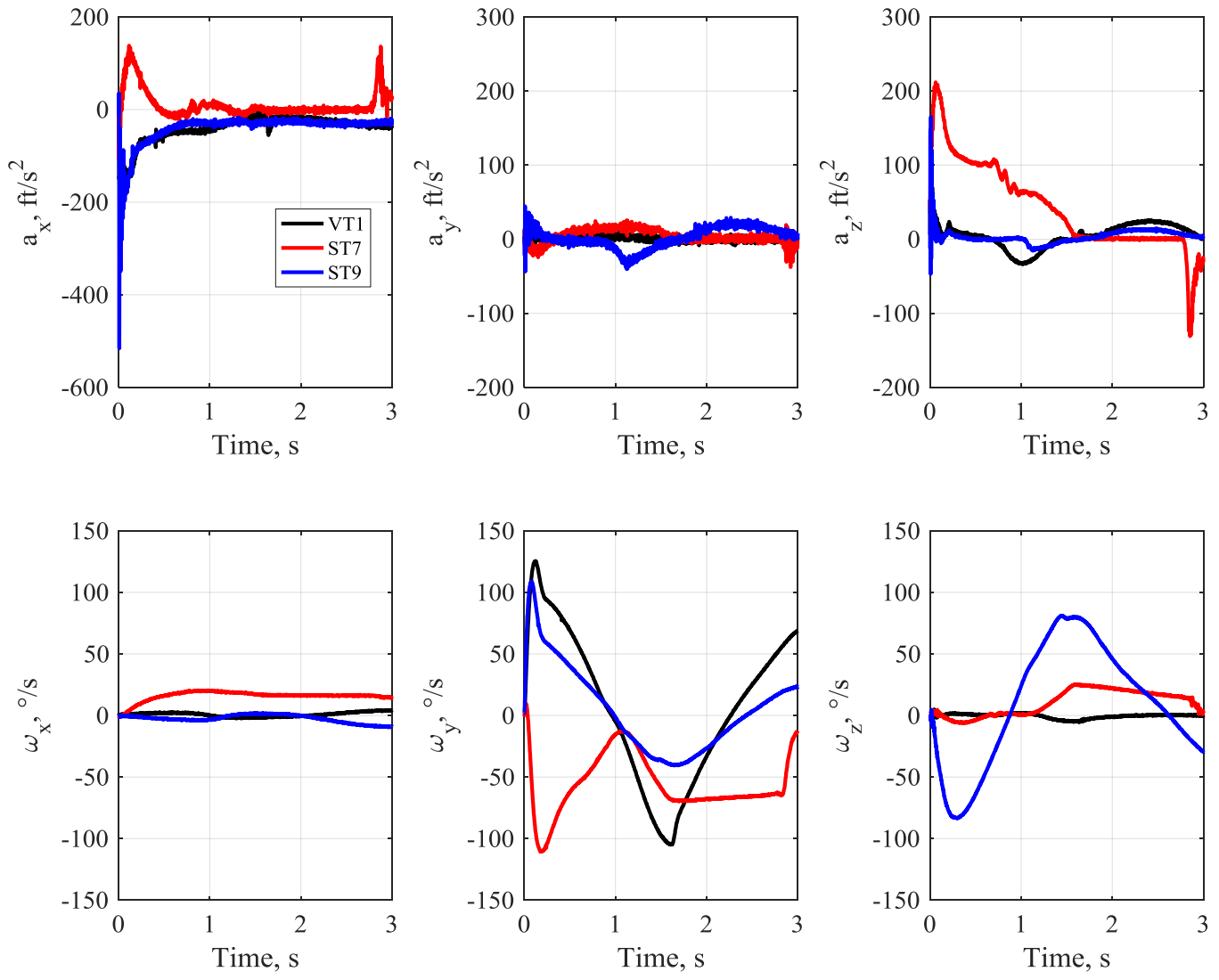

Figure 4. Smoothed body acceleration and rate data. 


\section{Post-Flight Trajectory Reconstruction}

This section provides descriptions of the techniques used to obtain the post-flight trajectory solutions and uncertainties.

\section{A. Initial Conditions and Photogrammetry}

Initializing the trajectory reconstruction and analyzing the post-impact behavior of the vehicle required accurate knowledge of the impact state conditions (position, velocity, and attitude) of the GTA in each WIT. To enable analysis of the impact conditions, two video cameras were positioned along the south side of the HIB. The GTA was outfitted with arrays of photogrammetry targets on three sides, shown in Figure 5(a), to accommodate tests with roll angles of $0^{\circ}, 90^{\circ}$, and $180^{\circ}$. An array of background targets was positioned along the northern edge of the HIB to define the global photogrammetry coordinate system as shown in Figure 5(b). These targets were designed to be easily discernable in images and video taken of the GTA during a WIT. Three-dimensional position time histories of each target were then determined using images from the cameras and PONTOS Live software. ${ }^{7}$ These target time history data were then in turn used to determine the GTA state at impact. ${ }^{4,5}$

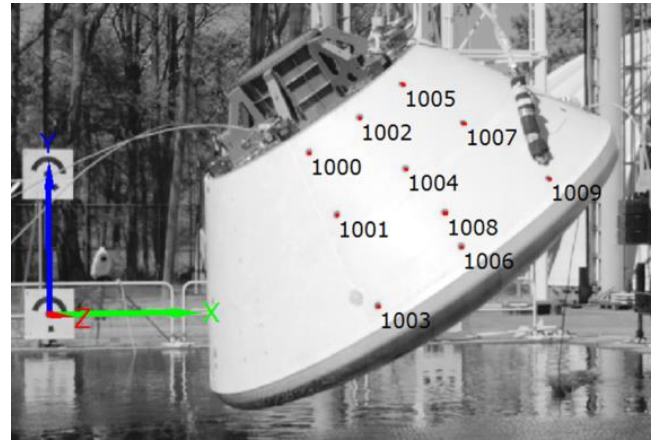

(a)

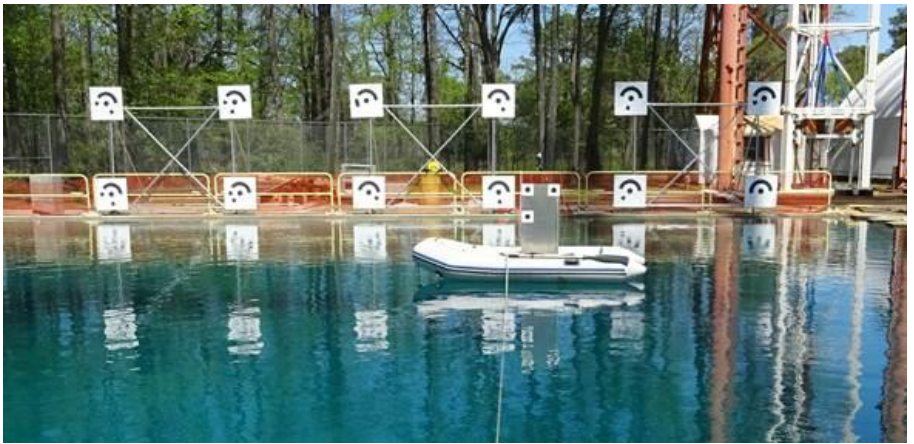

(b)

Figure 5. (a) GTA Photogrammetry Targets. (b) Global Coordinate System Photogrammetry Targets.

The local GTA coordinate system for any given camera frame was determined based on vectors between the photogrammetry target locations. The velocity was determined at Target 1006 representing the GTA CG projected onto the side of the backshell facing the cameras. This same location was used to define the velocity for the LS-DYNA model. The photogrammetry targets have a diameter of three inches. This, together with the precision of the alignment of the targets, affects the precision of the photogrammetry solution. Table 2 lists the initial condition $1 \sigma$ uncertainties due to photogrammetry precision used in the present analysis. The same value was used for $x-y-z$ components.

Table 2. Impact condition uncertainties, $1 \sigma$.

\begin{tabular}{cc}
\hline \hline Initial Condition & Uncertainty, 10 \\
\hline Position $(\mathrm{ft})$ & 0.0042 \\
Velocity (ft/s) & 0.0833 \\
Attitude angle (deg) & 0.1500 \\
\hline \hline
\end{tabular}

The test matrix with impact conditions of each test is listed in Table 3. These values are the observed, rather than targeted, conditions, obtained from photogrammetry. The rotation angles are ordered yaw-pitch-roll. ST5 and ST9 are initially rolled $180.0^{\circ}$ and $-90.0^{\circ}$, respectively. Note that some data were not available for ST7 due to equipment malfunction, so only pitch angle and vertical and horizontal velocities are listed. 
Table 3. Observed water impact conditions obtained from photogrammetry.

\begin{tabular}{cccccccc}
\hline \hline Test & Type & $\begin{array}{c}\text { Roll } \\
\text { (deg) }\end{array}$ & $\begin{array}{c}\text { Pitch } \\
\text { (deg) }\end{array}$ & $\begin{array}{c}\text { Yaw } \\
\text { (deg) }\end{array}$ & $\begin{array}{c}\text { Vertical } \\
\text { velocity } \\
(\mathbf{i n} / \mathbf{s})\end{array}$ & $\begin{array}{c}\text { Lateral } \\
\text { velocity } \\
(\mathbf{i n} / \mathbf{s})\end{array}$ & $\begin{array}{c}\text { Horizontal } \\
\text { velocity } \\
(\mathbf{i n} / \mathbf{s})\end{array}$ \\
\hline VT1 & Vertical & 1.20 & -35.08 & 2.23 & 387.37 & 9.44 & -1.91 \\
VT2 & Vertical & -6.24 & -45.91 & 0.76 & 401.62 & -0.91 & 0.34 \\
VT3 & Vertical & -3.19 & -24.11 & 0.45 & 387.44 & 0.05 & -0.25 \\
VT4 & Vertical & -1.44 & -24.00 & 0.46 & 381.29 & 1.04 & -1.27 \\
ST5 & Swing & -0.92 & 26.50 & -0.05 & 415.91 & -0.02 & -228.80 \\
ST6 & Swing & -1.56 & -18.51 & 0.07 & 341.74 & 2.89 & -448.50 \\
ST7 & Swing & 0.00 & -43.76 & 0.00 & 499.64 & 0.00 & -687.19 \\
ST8 & Swing & 28.47 & -43.51 & 0.78 & 326.41 & -2.03 & -648.56 \\
ST9 & Swing & 0.43 & 0.82 & -24.89 & 372.87 & -1.09 & -280.87 \\
VT10 & Vertical & -3.19 & -16.16 & 0.75 & 387.41 & 1.61 & -1.03 \\
\hline \hline
\end{tabular}

Figure 6 shows selected still frames from video recorded during ST7. In the left image, the camera is facing northward and the GTA is shown just prior to water impact, with the steep pitch angle evident. The center image shows an underwater angle also facing northward, with the GTA oriented almost perfectly horizontal. It is estimated that the leading edge of the heatshield came within several inches of the HIB floor. The right image shows a frame from a high-speed camera facing westward (downrange) just after the GTA rose up out of the water, and just prior to the second impact. Subsequent frames show the GTA almost completely obscured by the water splash, emphasizing the need to analyze reconstruction results.

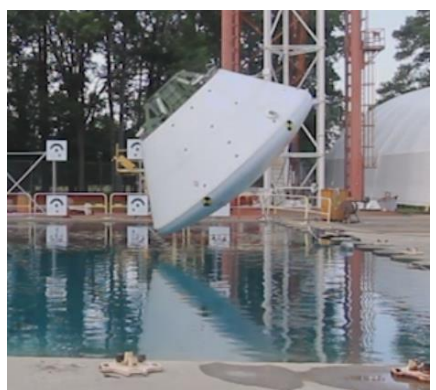

(a)

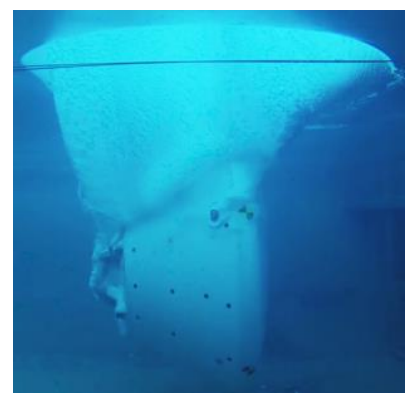

(b)

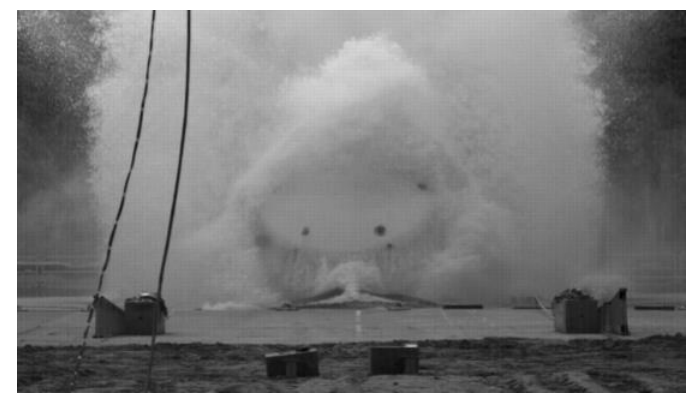

(c)

Figure 6. Selected still frames from flight video recorded during ST7. (a) GTA just prior to water impact. (b) GTA at maximum plunge depth. (c) GTA after rising out of the water and prior to secondary impact. Visible black markers are near outer edge of heatshield.

\section{Reconstruction}

The New Statistical Trajectory Estimation Program (NewSTEP) is a trajectory reconstruction tool that was developed initially for the Hyper-X program ${ }^{8}$ and has subsequently been utilized for a wide variety of launch vehicle and entry vehicle flight test programs, such as the recent application to the Low Density Supersonic Decelerator project. ${ }^{9}$ It draws from the basic approach of the original Statistical Trajectory Estimation Program (STEP) program developed in the 1960s. ${ }^{10}$ NewSTEP has the capability to perform pure inertial reconstruction based on direct numerical integration of the IMU linear accelerations and angular rates, or to perform a statistical reconstruction by processing these data along with other redundant data such as radar using a Kalman filter. The pure inertial reconstruction process was used for the GTA WIT reconstruction.

NewSTEP integrates the equations of motion of the vehicle expressed in a coordinate system relative to an oblate, rotating planet, using strapdown IMU measurements of angular velocity and linear acceleration to provide the kinematics. Gravitational accelerations are modeling using a J2 gravity field. A Fourth-Order fixed step Runge-Kutta algorithm is used to numerically integrate the equations of motion at a rate of $200 \mathrm{~Hz}$. NewSTEP is also capable of integrating the covariance matrix to produce uncertainty estimates for the reconstructed states, based on initial condition uncertainties and sensor specifications. 
The outputs of the process include three components of position and velocity, and a quaternion relating the body attitude to inertial space. The outputs were post-processed to transform them into appropriate coordinate frames for comparison with photogrammetry and simulation results.

\section{E. Visualization}

It is often of interest to visually represent the state time history of a vehicle. Visualization can facilitate understanding and analysis of vehicles exhibiting dynamic motion, as is the case in the GTA WIT. To that end, the Exploration Visual Environment (EVE) software was used to produce animations of the GTA reconstructed trajectory. EVE utilized the GTA state data (position, velocity, and attitude) in conjunction with a three-dimensional CAD model of the GTA to provide a virtual environment in which the user may view the trajectory from any angle. Geometry of the HIB was used to provide a reference for impact conditions.

\section{Results}

Trajectory reconstructions using NewSTEP as described in the preceding sections were performed for all drop tests and compared to results from flight video. Three seconds of reconstructed trajectory are shown here, since for some tests the state drift due to integration of sensor errors resulted in poor agreement with flight video after several seconds (recall that the onboard sensors were not specifically selected for use in trajectory reconstruction). This was considered sufficient for the purposes of the WIT since the primary interest was in the vehicle dynamics just after water impact. For the purposes of the present paper, selected trajectory reconstruction results from VT1, ST6, and ST7 are shown in Figure 7, and associated uncertainties are shown in Figure 8. The GTA attitudes are represented as 3-21, yaw-pitch-roll Euler angles that transform from the inertial frame to the body frame.

Several general observations are notable in the three seconds of reconstructed trajectory. Though the GTA does not achieve a steady, final state in this time, the Down position shows the position damping to a value close to zero (though not exactly zero since the GTA CG is just above the HIB waterline). The Northward position and velocity is negligible. The Westward position drift (negative East) is more pronounced in the ST cases than the VT, consistent with the additional initial Westward velocity. The reconstructed attitude will be discussed later.

ST7 was notable in that the targeted impact conditions were not met, resulting in the GTA entering the HIB at a shallower angle than planned. This in turn caused the GTA to plunge deeper and rise up out of the HIB farther than expected. A second impact, occurring when the GTA backshell impacted the water, imparted significant loads on the GTA structure (recall the second spike in acceleration just prior to $3 \mathrm{~s}$ in Figure 4). Additionally, due to technical difficulties, the 3D photogrammetry cameras failed to trigger for ST7. For that test, a similar 2D photogrammetry process was used with the north-south positions treated as constant. ST7 Down position is the highest of the three selected tests, which is again consistent with the flight video.

For all WIT reconstruction results shown here, the position and velocity uncertainties increase across time as sensor errors accumulate during integration. The $3 \sigma$ uncertainties are shown in Figure 8. Also note the larger out-ofplane (Northward) position and velocity uncertainties seen in ST7, a result of the lack of out-of-plane photogrammetry on that WIT. The large spikes in attitude uncertainties are occur when the GTA approaches Euler angle singularities (this does not affect the reconstruction since NewSTEP integrations the quaternions form of the attitude equations). 

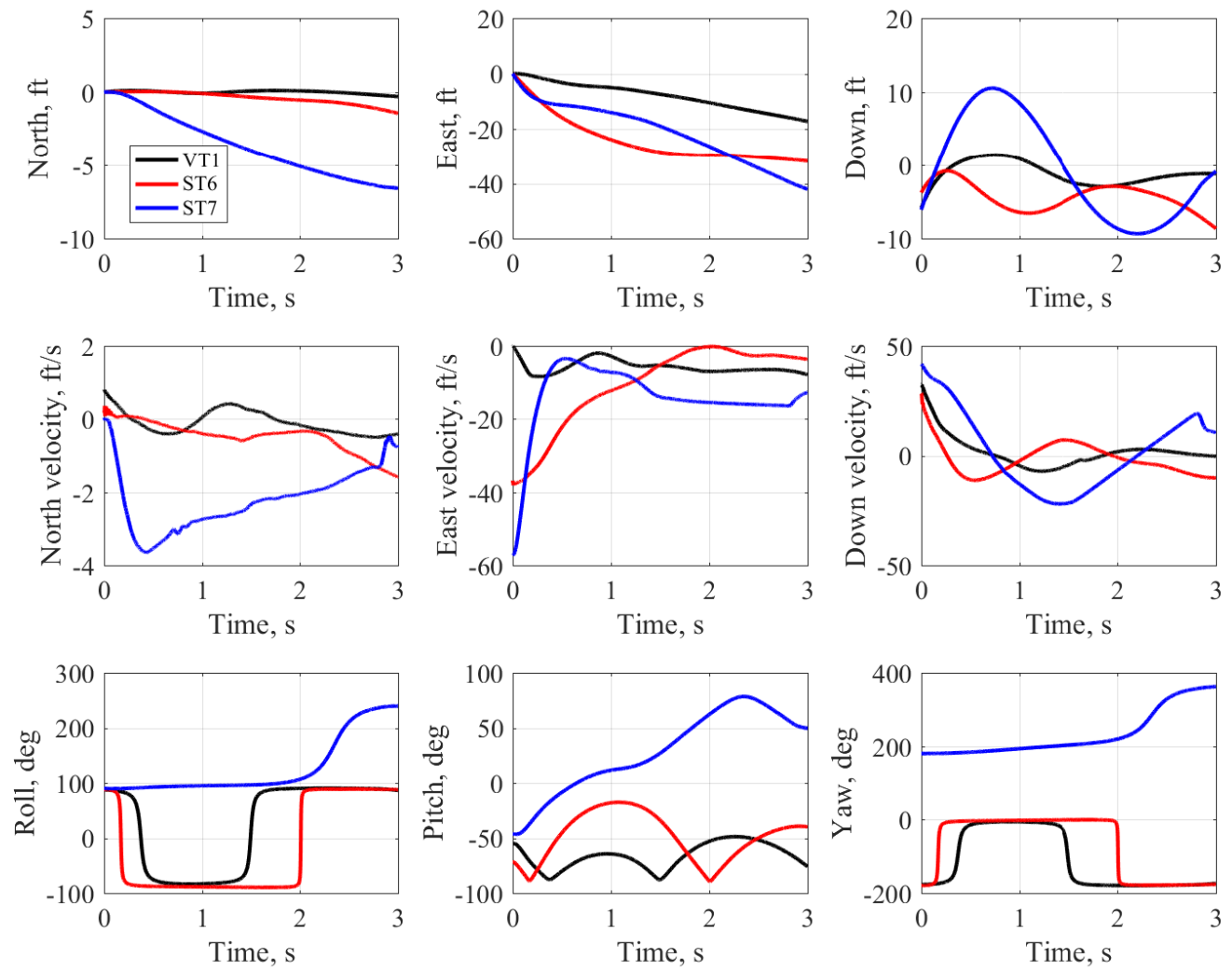

Figure 7. Reconstructed GTA position, velocity, and attitude.
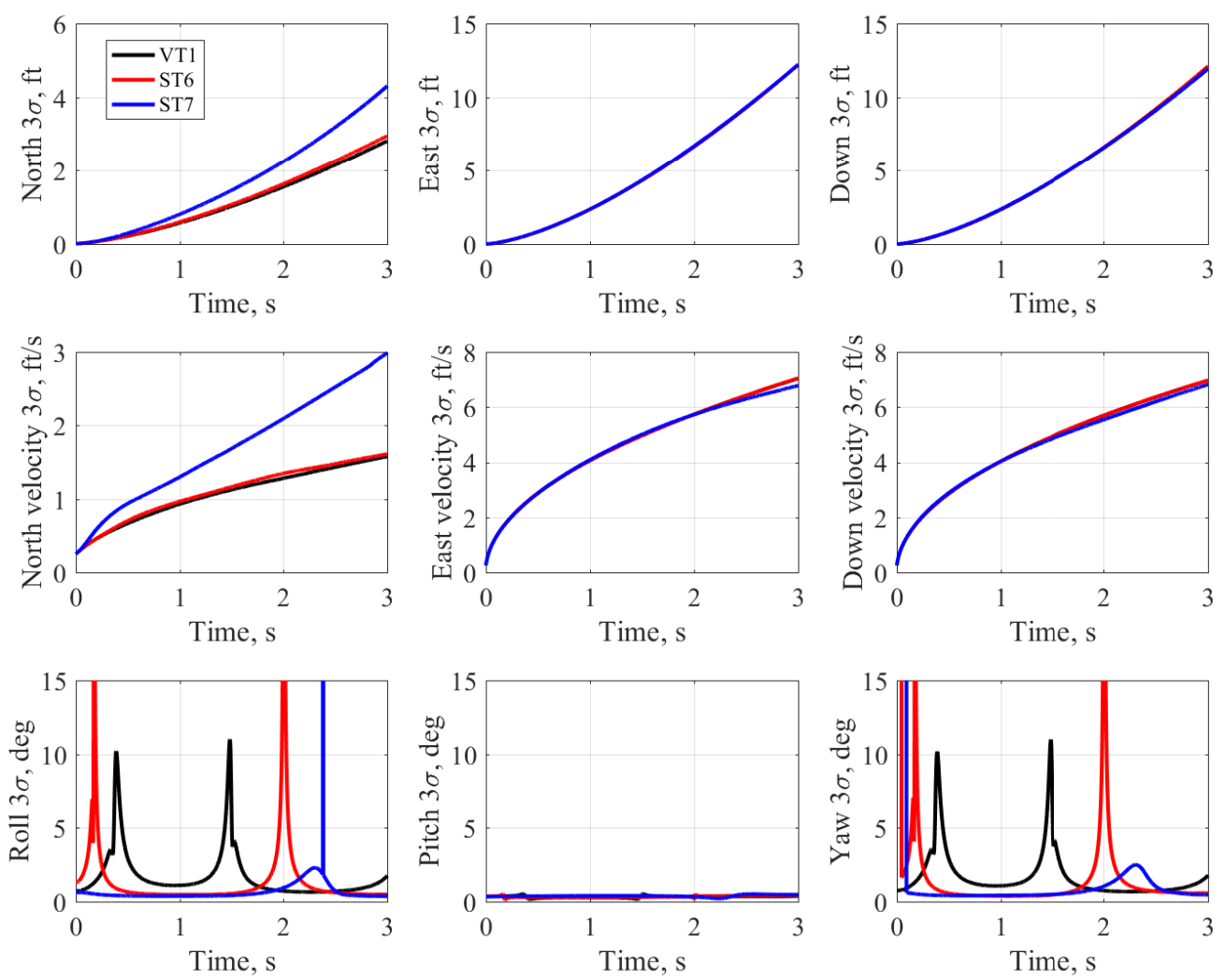

Figure 8. Reconstructed GTA position, velocity, and attitude $3 \sigma$ uncertainties.

8

American Institute of Aeronautics and Astronautics 
The Euler angle representation of the vehicle attitudes in Figure 7 and Figure 8 is not particularly intuitive, especially for ST7 since the GTA flipped upside down during that test. However, it can be observed that VT1 and ST6 exhibited sinusoidal pitching motion, with ST6 reaching a significantly higher pitch angle, consistent with the flight video. It is more constructive to visually compare the reconstructed attitudes with the flight video rather than to interpret Euler angle representations. Figure 9 shows selected still frames from the reconstructed ST7 trajectory visualized in EVE. Similar visualizations were created using the reconstructed trajectories for the other WIT.

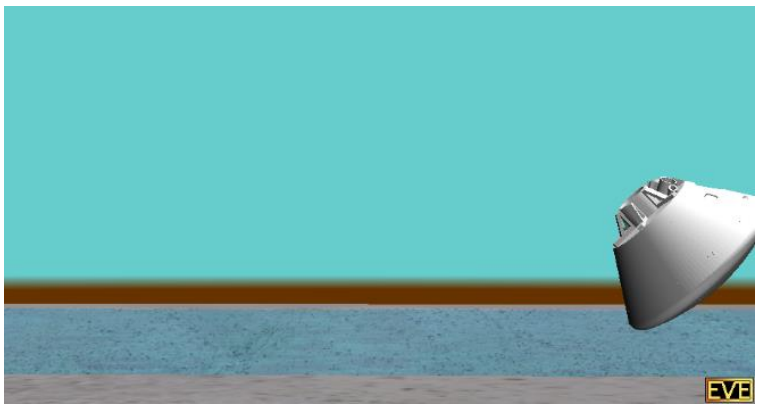

(a)

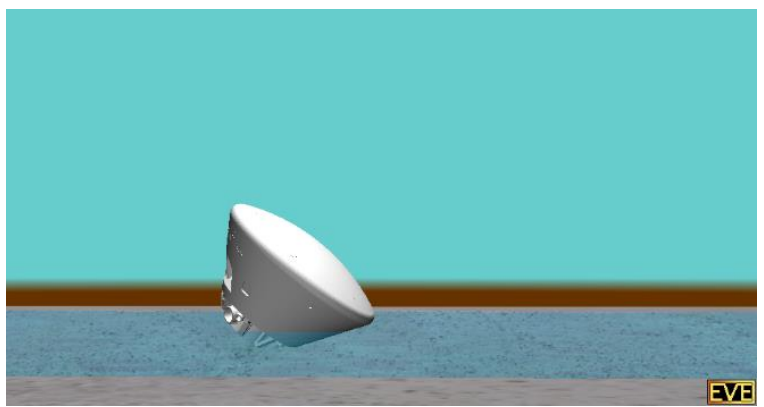

(c)

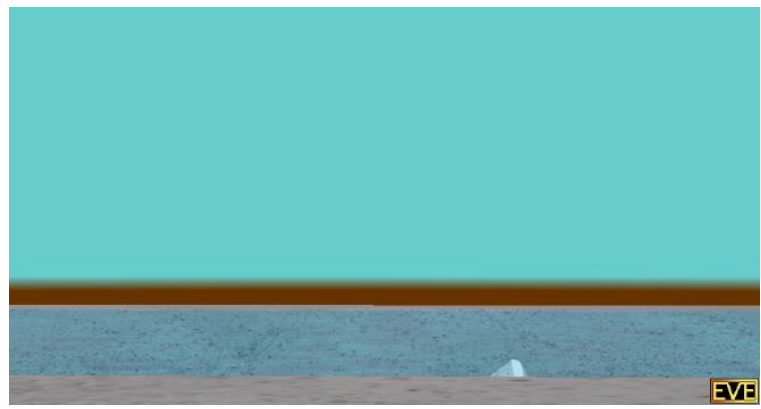

(b)

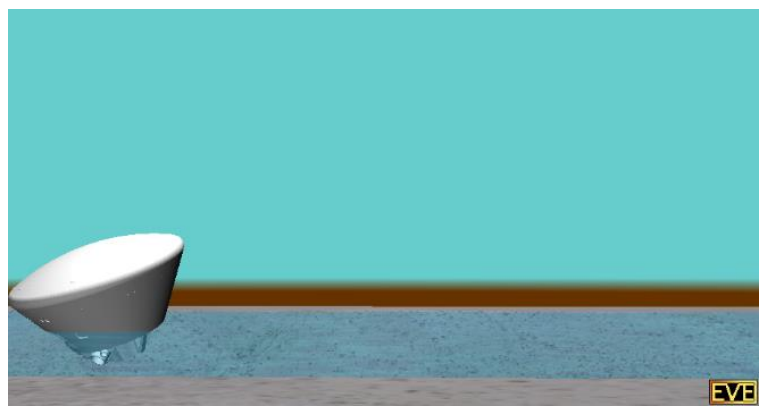

(d)

Figure 9. Selected still frames in sequential order from ST7 reconstructed trajectory visualized in EVE. (a) GTA just prior to water impact. (b) GTA at maximum plunge depth. (c) GTA rising up out of the HIB. (d) Second impact.

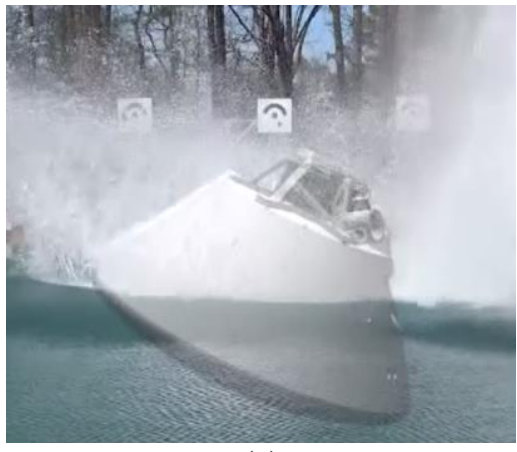

(a)

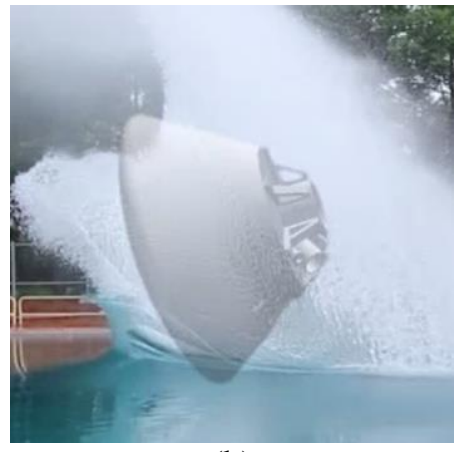

(b)

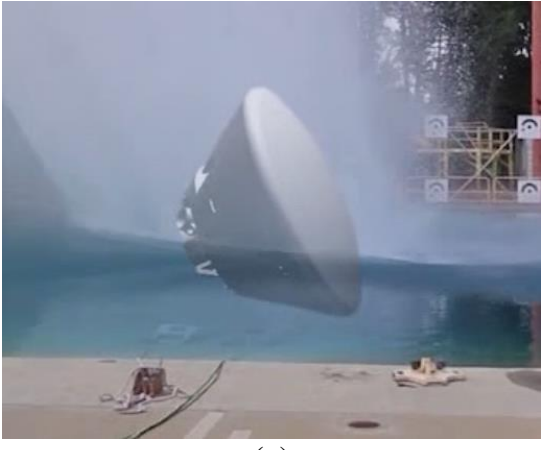

(c)

Figure 10. Still frames from (a) VT1, (b) ST6, and (c) ST7 flight videos with GTA geometry driven by reconstructed trajectory data in EVE. Note that the GTA visible in these frames is a CAD model superimposed on the video frame, and the actual GTA is obscured by the water splash.

The EVE animations of the reconstructed trajectories were compared directly against flight video recorded during the WIT by adjusting the camera in EVE to match the angle in the video, recording an animation, and then superimposing the animation over the flight video. Figure 10 shows a still frame from the VT1, ST6, and ST7 flight videos with GTA geometry driven by reconstructed trajectory data in EVE superimposed on the frame. Though there was lack of information on the physical position and view angle of the actual camera, resulting in the overlays drifting 
as in Figure 10(b), these images illustrate how visualization of the vehicle can be useful, since at these points in the trajectory the GTA is almost totally obscured by the water splash. It can also be used to validate the attitude reconstruction, since again the Euler angle representation can be difficult to interpret directly.

\section{Summary and Conclusions}

Reconstructed trajectory states and associated uncertainties for a series of Orion Ground Test Article water impact tests at the NASA Langley Research Center Hydro Impact Basin have been presented. Both vertical and swing tests designed to simulate off-nominal impact conditions were investigated. Though the sensors were not specifically designed for use in trajectory reconstruction, the reconstructed trajectories agreed well with flight video for the first three seconds after impact, which satisfied the WIT goals of trajectory comparison just after water impact.

\section{Acknowledgements}

The authors thank Scott Angster (AMA, Inc.) for EVE analysis and support, Eric Fay (AMA, Inc.) for generating HIB geometry for use in EVE, and Bob Evangelista (AMA, Inc.) for producing videos overlaying EVE trajectory reconstruction animations over flight video.

\section{References}

${ }^{1}$ Norris, S. D., Marshall, P. F., Cichan, T., McNamara, R., and Cox, B., "Orion: Lessons Learned from EFT-1 and, EM-1, AA2, and EM-2 status," AIAA SPACE Forum, Long Beach, CA, September 2016, AIAA 2016-5416.

${ }^{2}$ Huot, D., "The Ins and Outs of NASA's First Launch of SLS and Orion," NASA, [online article], 27 November 2015, URL: https://www.nasa.gov/feature/the-ins-and-outs-of-nasa-s-first-launch-of-sls-and-orion [cited 30 May 2017].

${ }^{3}$ Hambleton, K., "NASA Affirms Plan for First Mission of SLS, Orion," NASA, [online article], 12 May 2017, URL: https://www.nasa.gov/feature/nasa-affirms-plan-for-first-mission-of-sls-orion [cited 30 May 2017].

${ }^{4}$ Littell, J. D., "Large Field Photogrammetry Techniques in Aircraft and Spacecraft Impact Testing," Society of Experimental Mechanics 2010 Annual Conference Proceedings, Indianapolis, June 7-10, 2010, pp. 55-67.

${ }^{5}$ Vassilakos, G. J., and Mark, S. D., "Orion Ground Test Article Water Impact Tests: Photogrammetric Evaluation of Impact Conditions," NASA Contractor Report, September 2017.

${ }^{6}$ Hallquist, J. O., "LS-DYNA Theoretical Manual,” LSTC Livermore, 1998.

7“PONTOS Live," GOM, [online article], URL: http://www.gom.com/metrology-systems/pontos-live.html [cited 1 June 2017].

${ }^{8}$ Karlgaard, C. D., Tartabini, P. V., Blanchard, R. C., Kirsch, M., and Toniolo, M. D., "Hyper-X Post-Flight Trajectory Reconstruction," Journal of Spacecraft and Rockets, Vol. 43, No. 1, 2006, pp. 105-115.

${ }^{9}$ Karlgaard, C. D., O’Farrell, C., Ginn, J. M., and Van Norman, J. W., "Supersonic Flight Dynamics Test 2: Trajectory, Atmosphere, and Aerodynamics Reconstruction," American Astronautical Society, AAS Paper 16-217, February 2016. 1970.

${ }^{10}$ Wagner, W. E. and Serold, A. C., "Formulation on Statistical Trajectory Estimation Programs," NASA CR-1482, January 